to appear in: Annals of the New York Academy of Sciences

\title{
Collisionless Relaxation in Galactic Dynamics and the Evolution of Long Range Order
}

\author{
HENRY E. KANDRUP尚 \\ Department of Astronomy and Department of Physics \\ and Institute for Fundamental Theory \\ University of Florida, Gainesville, Florida 32611
}

\begin{abstract}
This talk presents a critical assessment of certain aspects of collisionless galactic dynamics, focusing on the interpretation and limitations of the collisionless Boltzmann equation and the physical mechanisms associated with collisionless, or near-collisionless, relaxation. Numerical and theoretical arguments are presented to motivate the idea that the evolution of a system far from equilibrium should be interpreted as involving nonlinear gravitational Landau damping, a picture which implies a greater overall coherence and remembrance of initial conditions than is implicit in the conventional paradigm of violent relaxation.
\end{abstract}

\section{INTRODUCTION AND MOTIVATION}

The principal aim of the work described here is to understand the gravitational $N$-body problem for a collection of nearly point "particles" of comparable mass, such as, for example, the stars in a galaxy. This $N$-body problem is not directly applicable for problems involving galaxy formation, or in understanding the structure and evolution of spiral galaxies, where dissipative gas dynamics must play an important role. However, it should be relevant in understanding the structure of elliptical galaxies, gas-poor ${ }^{1}$ objects which are typically assumed to be in or near a (meta-)equilibrium. ${ }^{2}$ It should, moreover, be important in understanding the evolution of elliptical galaxies when, as must often occur, ${ }^{3}$ they are displaced from their near-equilbrium by a collision or other close encounter with some companion object.

${ }^{a}$ HEK was supported in part by National Science Foundation Grant No. PHY92-03333. Some of the numerical calculations were facilitated by computer time provided by IBM through the Northeast Regional Data Center (Florida). This article is based in part on collaborations with Elaine Mahon, Christos Siopis, and Barbara Eckstein, students at the University of Florida who have been supported by NASA through the Florida Space Grant Consortium. 
The conventional wisdom of galactic dynamics ${ }^{2}$ asserts that, over sufficiently short time scales, the structure and evolution of a collection of $N$ self-gravitating point masses can be described by the collisionless Boltzmann equation, the gravitational analogue of the VlasovPoisson system of plasma physics. Alternatively, it is assumed that, on longer time scales, one must allow for discreteness effects, described by the collisional Boltzmann, or FokkerPlanck equation, the latter being simply the gravitational analogue of the Landau equation from plasma physics.

In this context, it is typically assumed (cf. Ref. [4]) that there are four distinct sorts of "relaxation" processes, namely (1) "violent relaxation," (2) gravitational Landau damping, (3) phase mixing, and (4) "collisional relaxation." Violent relaxation ${ }^{2,5}$ refers to the early stages of evolution in which a system, initially far from any steady state, evolves towards some meta-equilibrium, i.e., a time-independent solution to the collisionless Boltzmann equation $(C B E)$. By contrast, Landau damping and phase mixing reflect smaller amplitude effects acting in a system which is much closer to a meta-equilibrium. Collisional relaxation is associated with discreteness effects which, dating back to Chandrasekhar, ${ }^{6}$ have typically been modeled as an incoherent sum of binary encounters, i.e., gravitational Rutherford scattering.

The first three of these mechanisms, incorporated in the $C B E$, are presumed to induce an efficient approach towards some meta-equilibrium on a time scale of order $t_{c r}$, a characteristic crossing time for the system in question. By contrast, collisional relaxation is presumed to be important only on a much longer relaxation time $t_{R}$ which, for systems as large as an entire galaxy, is orders of magnitude longer than $t_{H}$, the age of the Universe. In the language of statistical physics, one thus assumes that a mean field theory, based on the $C B E$, accurately describes the structure and evolution of a gas-poor elliptical galaxy over time scales shorter than or comparable to the age of the Universe.

In this talk, I would like to play devil's advocate and ask to what extent one can really be confident that this conventional wisdom is completely correct. Specifically, this talk will address four distinct questions: (1) Does one really know that the $C B E$ is the whole story? (2) In what sense, and to what extent, does the $C B E$ imply an evolution towards some meta-equilibrium? (3) How should one interpret the flow associated with the $C B E$ and, related to this, what is the dynamical principle that determines the form of the metaequilibrium towards which an $N$-body system is presumed to evolve? (4) What precisely is the connection between the $C B E$ and the $N$-body problem, and, in particular, what do $C B E$ characteristics computed for a smooth initial distribution function have to do with orbits generated as a solution to the full gravitational $N$-body problem?

The principal substantive conclusion is that, contrary to what is usually assumed by galactic dynamicists, phase mixing, Landau damping, and violent relaxation should be viewed as (not so different) manifestations of a single phenomenon. As is well known to plasma physicists, linear Landau damping is in fact a type of phase mixing. Moreover, numerical experiments and theoretical considerations suggest that the evolution of a system 
far from equilibrium can be interpreted fruitfully as entailing nonlinear Landau damping, this implying a greater overall coherence and remembrance of initial conditions than is implicit in the conventional picture of violent relaxation.

\section{IS THE COLLISIONLESS BOLTZMANN EQUATION THE WHOLE STORY?}

The basic assumption underlying the $C B E$ is that the system in question can be described probabilistically in terms of a smooth one-particle distribution function, $f\left(x^{a}, v_{a}, t\right)$, which can be interpreted as a phase space number or mass density that yields the probability of finding a particle near the spatial point $x^{a}$ with velocity $v_{a}$ at time $t$. (In what follows, it will be assumed that each particle has a mass $m=1$ since, in the context of a mean field theory, allowing for a distribution of particle masses has absolutely no effect.) In the context of the initial value problem, this implies that the true initial distribution function, given as a sum of $N$ phase space delta functions, has been replaced by a smooth function $f\left(x^{a}, v_{a}, 0\right)$.

The $C B E$ manifests the idea that the distribution function $f$ will free stream in the self-consistent potential $\Phi$ associated with $f$, so that

$$
\frac{d f}{d t} \equiv \frac{\partial f}{\partial t}+v^{a} \frac{\partial f}{\partial x^{a}}-\frac{\partial \Phi}{\partial x^{a}} \frac{\partial f}{\partial v_{a}}=0,
$$

where

$$
\nabla^{2} \Phi\left(x^{a}, t\right)=4 \pi G \int d^{3} v f\left(x^{a}, v_{a}, t\right) .
$$

In the context of a many-particle phase space description, this is equivalent to assuming that the two-particle distribution function $g(1,2)$ factorises into a product of one-particle $f$ 's, so that $g(1,2) \approx f(1) f(2)$, i.e., neglecting entirely the effects of particle-particle correlations. It is assumed that this neglect is justified on time scales short compared with the natural time scale $t_{R}$ associated with gravitational Rutherford scattering, a time scale which, for very large $N$, is much larger than $t_{c r}$.

Unfortunately, this neglect of particle correlations constitutes an uncontrolled approximation. Thus, for example, unlike the case of a neutral plasma, one cannot derive the next order correction to the $C B E$ in the context of some systematic perturbation expansion. Physically, the problem is that there is no shielding to vitiate the long range $1 / r^{2}$ force. This is, e.g., manifested by the fact that a $1 / r$ potential yields an infinite cross section, so that, when evaluating the effects of binary encounters in the usual way for an infinite and

\footnotetext{
${ }^{b}$ It is ironic that Lynden-Bell's original paper $^{5}$ on violent relaxation does not implement clear cut distinctions amongst violent relaxation, Landau damping, and phase mixing. Rather, it suggests a more integrated approach which views phase mixing-cum-Landau damping as an important part of violent relaxation, an approach more consistent, in some respects, with the ideas advocated in this paper than with conventional wisdom.
} 
homogeneous system, one encounters logarithmic divergences in the limit of large impact parameter (the problematic Coulomb logarithm of galactic dynamics).

Physically, one might hope to circumvent this difficulty by first identifying the bulk mean field force acting at any given point in space and then treating "fluctuations away from mean field conditions" as the source of deviations from mean field theory. For $N \gg 1$ one might expect that these fluctuations are small, so that their effects do in fact constitute a small perturbation. This splitting into mean field plus fluctuations can be introduced formally, e.g., by using time-dependent projection operators, ${ }^{7}$ but is difficult to implement concretely because of the apparent absence of a clean separation of time scales.

One case where one can try to perform a concrete calculation is for the toy model of a system that is rotating with an angular velocity $\Omega$ carefully chosen to ensure that the mean field equilibrium is homogeneous (here the rotation provides a fictitious force that plays the role of the uniform oppositely charged background in a plasma). In this case, one finds that the screened potential $\exp (-\kappa r) / r$ of plasma physics is replaced by an oscillatory potential $\exp (i \kappa r) / r$, this reflecting the fact that an homogeneous system is unstable towards sufficiently long wavelength perturbations (the Jeans instability). The conventional response is to introduce by hand a cut off at a length scale $R_{\text {sys }}$ comparable to the size of the system, supposing that the system was generated at some earlier time by the fragmentation of a much larger, nearly homogeneous entity that experienced the Jeans instability.

As demonstrated by Thirring and coworkers (cf. Ref. [8]), there does in fact exist a well defined thermodynamic limit in which gravitational mean field theory becomes exact. However, this limit is an unusual one. Given that realistic self-gravitating systems are inhomogeneous, one cannot simply introduce the ordinary thermodynamic limit, with number $N \rightarrow \infty$ and volume $V \rightarrow \infty$ but finite density $n=N / V$. Rather, one must instead allow for a limit in which the coupling constant $G$ also scales. The problem, however, is that there is no guarantee that this limit is physically realistic and that, even assuming that it is physical, there are no rigorous (or even quasi-rigorous) estimates as to the time scale $t_{R}(N)$ on which the mean field description would be expected to fail.

Given that theoretical analyses have as yet proven inconclusive, one might instead seek recourse to numerical experiments. This, however, is difficult. For generic systems not characterised by a high degree of symmetry, the $C B E$ is a partial differential equation in six independent phase space variables which is extremely expensive to solve computationally. Moreover, the time required to solve the $N$-body problem using an honest direct summation code scales as $N^{2}$, so that the consideration of very large $N$, where the $C B E$ is expected to be valid, becomes prohibitive. The approximately validity of the $C B E$ has been corroborated in the sense that $N$-body realizations of meta-equilibria often appear to behave stably for relatively short time scales, and that, at least for short time scales, various velocity moments extracted from $N$-body simulations and time-dependent solutions to the $C B E$ yield no gross discrepancies. However, computational limitations have prevented truly detailed 
comparisons of $N$-body and $C B E$ evolutions hitherto.

There is in fact one concrete setting where detailed computations have been done, namely the toy model of one-dimensional gravity, i.e., a collection of infinite plane sheets characterized by a two-body potential ${ }^{9}$

$$
V\left(\left|x_{i}-x_{j}\right|\right)=G\left|x_{i}-x_{j}\right|
$$

For this simple model, the phase space is only two-dimensional and the forces are trivial computationally, so that it is relatively easy to perform highly accurate computations. The net result of such simulations is that, at least for times as long as $10^{2}-10^{3} t_{c r}$, there is a reasonably good overall agreement between $N$-body simulations and an evolution governed

by the $C B E .{ }^{10}$ Interestingly, however, the evolution seems to be substantially more complex than what most astronomers would expect for three-dimensional gravity (cf. Refs. [1112]). These conclusions seem highly suggestive. However, they must both be taken with a fair grain of salt since there is no compelling reason to believe that the evolution of one-dimensional gravitational systems is qualitatively similar to the evolution of "real" three-dimensional systems.

In summary, even though a mean field theory based on the $C B E$ may seem well motivated physically, there is as yet no rigorous proof of its validity and, in particular, no rigorous estimate as to the time scale on which it might be expected to fail. Moreover, as discussed below, there are reasons to suspect that even very small corrections could be important by accelerating the approach towards equilibrium and, especially, by serving to "fuzz out" small scale structures predicted by the $C B E$ to arise as a self-gravitating system approaches some meta-equilibrium.

\section{IN WHAT SENSE DOES THE COLLISIONLESS BOLTZMANN EQUATION IMPLY AN APPROACH TOWARDS EQUILIBRIUM?}

In addressing the question of evolution, the most important thing to recognise about the $C B E$ is that it, like the true $N$-body problem, is Hamiltonian. Specifically, the $C B E$ constitutes a non-canonical Hamiltonian system, formulated in an infinite-dimensional phase space, where the fundamental dynamical variable is the distribution function $f$ itself. ${ }^{13,14}$ The proof that the $C B E$ is Hamiltonian entails the identification of a Hamiltonian functional $\mathcal{H}[f]$ and a Lie bracket $[.$, . $]$ defined on functionals $\mathcal{A}[f]$ and $\mathcal{B}[f]$, so chosen that the $C B E$ takes the form

$$
\frac{\partial f}{\partial t}+[\mathcal{H}, f]=0 .
$$

It is straightforward to verify that the antisymmetric operation

$$
[\mathcal{A}, \mathcal{B}]=\int d^{3} x d^{3} v f\left\{\frac{\delta \mathcal{A}}{\delta f}, \frac{\delta \mathcal{B}}{\delta f}\right\}
$$

with $\delta / \delta f$ a functional derivative and $\{.,$.$\} the ordinary Poisson bracket of particle me-$ chanics, defines a bona fide Lie bracket acting in the infinite-dimensional phase space of 
distribution functions. However, if one identifies $\mathcal{H}[f]$ as the mean field energy, i.e.,

$$
\mathcal{H}[f]=\frac{1}{2} \int d^{3} x d^{3} v v^{2} f\left(x^{a}, v_{a}, t\right)-\frac{G}{2} \int d^{3} x d^{3} v \int d^{2} x^{\prime} d^{3} v^{\prime} \frac{f\left(x^{a}, v_{a}, t\right) f\left(x^{\prime a}, v_{a}^{\prime}, t\right)}{\left|\mathbf{x}-\mathbf{x}^{\prime}\right|}
$$

it is easy to see that, with this bracket, eq. (4) reduces to the $C B E$ in the form

$$
\frac{\partial f}{\partial t}-\{E, f\}=0
$$

where $E=\frac{1}{2} v^{2}+\Phi[f]$ is the particle energy.

Associated with this Hamiltonian character is the existence of an infinite number of conserved quantities, the so-called Casimirs, which generalise the notion of conservation of phase (or Liouville's Theorem) for a distribution function $f$ evolving in an external potential. ${ }^{15}$ Specificially, one knows that, for any function $\chi(f)$, the numerical value of $C[f]=\int d^{3} x d^{3} v \chi(f)$ is independent of time. $[$ One concrete manifestation of these Casimirs is the fact that any phase-preserving perturbation of a $C B E$ equilibrium must be generated by a canonical transformation. ${ }^{16}$ Every time-independent equilibrium solution $f_{0}$ corresponds to an extremal point of the mean field energy, i.e., $\delta^{(1)} \mathcal{H} \equiv 0$ for all phase preserving perturbations of the equilibrium.

The Hamiltonian character of the evolution is crucial because it precludes entirely the possibility of any pointwise approach towards a time-independent equilibrium. Only by replacing the true distribution function $f$ by some coarse-grained $\bar{f}$ can one hope to identify an object which actually approaches equilibrium. Any meaningful discussion of "the approach towards equilibrium" must be formulated in the context of a coarse-grained distribution function or, alternatively, some set of coarse-grained observables constructed from the true distribution function by some phase space averaging.

Very little is known mathematically about an evolution governed by the $C B E$. Recently, however, it has been proven that the $C B E$ manifests global existence. ${ }^{17,18}$ Specifically, one knows that, given sufficiently smooth initial data $\left(C^{1+}\right)$, the distribution function will never diverge. In other words, unlike the case of a perfect fluid, the evolution never leads to caustics or shocks. This suggests in particular that smooth initial conditions cannot evolve near-singular cores or other lumpy exotica of the form discovered recently using the Hubble Space Telescope. ${ }^{19}$

Unfortunately, however, there are no rigorous results about a (suitably coarse-grained) approach towards equilibrium. Rather, the only results obtained to date relate to the asymptotic behaviour of time integrals of quantities like $\mathcal{K}[f]$ or $\mathcal{V}[f]$, the mean field kinetic and potential energies. Thus, e.g., in certain cases one can prove ${ }^{20}$ the existence of quantities like

$$
K \equiv \lim _{T \rightarrow \infty} \frac{1}{T} \int_{0}^{\infty} \mathcal{K}[f(t)] d t .
$$

${ }^{c}$ Significantly, however, even though the $C B E$ admits this infinite collection of conserved quantities, it is extremely unlikely that it is integrable (unlike, e.g., the Korteweg-de Vries equation). 
However it has not been possible to prove statements about the behaviour of (say) $\mathcal{K}[\bar{f}]$ at a fixed instant of time, let alone the behaviour of the full $\bar{f}$.

One might naively suppose that this simply reflects the fact that any such result would be very hard to prove. However this is not completely true. Specifically, there exist exact, time-dependent solutions to the $C B E$, corresponding to a system that remains bounded in space, that exhibit no approach towards a coarse-grained equilibrium. ${ }^{21,22}$ Rather, these solution correspond to finite amplitude, undamped oscillations about an otherwise timeindependent equilibrium $f_{0}$.

There is of course no reason to expect a priori that such oscillating solutions actually exist in nature. However, it appears that they can arise naturally at least in the context of one-dimensional gravity. ${ }^{10}$ Specifically, for both $N$-body simulations and the $C B E$, the evolution of counter-streaming initial conditions (two equilibria engaged in a head-on collision) can yield a final $f(t)$ which involves undamped oscillations about a time-independent $f_{0}$ which, significantly, contains "holes," i.e., regions in the middle of the occupied phase space region where $f_{0} \rightarrow 0$.

\section{HOW SHOULD ONE INTERPRET THE FLOW ASSOCIATED WITH THE COLLISIONLESS BOLTZMANN EQUATION?}

\section{"Violent Relaxation"}

Most discussions of the evolution of a self-gravitating system initially far from a metaequilibrium have been formulated within the context of the paradigm of violent relaxation, a sort of "self-consistent egg beater" model originally proposed by Lynden-Bell thirty years ago. ${ }^{5}$ Different workers have interpreted this paradigm in rather different ways. Indeed, the conventional wisdom would appear to ignore many of the fine points made in the original paper. However, (almost) all versions of violent relaxation seem to incorporate four basic ingredients:

1. Attention focuses on a coarse-grained distribution function $\bar{f}$ which can "fuzz out" in a way that is impossible for the true $f$, which is strongly constrained by conservation of phase. The obvious point here is that if $f$ satisfies the $C B E$, in general the coarsegrained $\bar{f}$ will not. Lynden-Bell starts from the assumption that the fundamental object of interest is a smooth one-particle $f$ that satisfies exactly the $C B E$ and then implements a coarse-graining of the one-particle phase space to extract an appropriate $\bar{f}$. However, many later workers have sought to relate $\bar{f}$ directly to the full $N$-particle dynamics, as derived, e.g., from numerical simulations. This difference in perspective is important. Lynden-Bell assumes that the $C B E$ is exact in some suitable $N \rightarrow \infty$ continuum limit and formulates his entire theory in that limit. For him, therefore, violent relaxation need say little if anything about individual particles, and should be interpreted instead as tracking a coarse-grained free streaming of phase space fluid 
elements.

2. One invokes the effects of a strongly time-dependent bulk potential $\Phi[f]$ to help "shuffle" the coarse-grained $\bar{f}$. The initial $f(0)$ yields the mass density $\rho(0)$ and, hence, the gravitational potential $\Phi(0)$ which determines the initial motion of each phase point in the system. In general, $f(0)$ will be far from equilibrium, so that $\Phi$ will induce changes in $f$ on a time scale $\sim t_{c r}$. However, changes in $f$ correspond in general to changes in $\Phi$, so that the potential itself will vary significantly on the same time scale $t_{c r}$. This implies in turn that the particle energy $E=\frac{1}{2} v^{2}+\Phi$ associated with any characteristic will be strongly time-dependent. In particular, if the potential exhibits large enough variations on a sufficiently short time scale, $E$ will be far from an adiabatic invariant, so that one might expect that the energies of the different characteristics will become "well shuffled" and/or randomized.

3. One supposes that, as a result of this convulsive process, $\bar{f}$ will evolve towards, albeit not necessarily to, the "most likely" $\bar{f}_{0}$ consistent with an appropriate set of constraints. Two different types of constraint are usually considered, namely (1) bulk holonomic constraints, such as conservation of energy and particle number, and (2) the residual, coarse-grained effects of conservation of phase, which may still prove important if the system is sufficiently dense. Although crucial conceptually, the latter are often unimportant as a practical matter (unless, e.g., the system is very dense) and, for that reason, will be ignored in the following.

4. One supposes further that the "most likely" $\bar{f}_{0}$ can be derived from a combinatoric argument by determining the macrostate corresponding to the largest number of microstates. To the extent that the constraints associated with conservation of phase are unimportant, this leads to the identification of the most likely $\bar{f}_{0}$ as being that particular coarse-grained distribution function that maximizes the Boltzmann entropy

$$
S[\bar{f}]=-\int d^{3} x \int d^{3} v \bar{f} \log \bar{f} .
$$

It is easily seen that, by combining these four ingredients, one is led to a predicted evolution towards an isothermal distribution of the form

$$
\bar{f}_{0} \propto \exp \left\{-\beta\left(v^{2} / 2+\Phi\left[\bar{f}_{0}\right]\right)\right\} .
$$

(Allowing for coarse-grained conservation of phase would modify eq. (9) to yield a degenerate isothermal.)

Lynden-Bell would stress that, albeit involving combinatoric arguments familiar from information theory, his derivation of eq. (10), which deals with phase space fluid elements rather than discrete particles, is not an entropy argument in the usual sense. One clear indication of this is the fact that the particle mass $m$ does not enter into $\bar{f}_{0}$. If one implements a "true" entropy argument for a collection of particles with a distribution of masses, one finds instead ${ }^{64}$ a mass-dependent extremal entropy configuration of the form $\bar{f}_{0} \propto \exp \left\{-\beta\left(m v^{2} / 2+m \Phi\left[\bar{f}_{0}\right]\right)\right\}$. 
The "prediction" of an isothermal was considered a triumph back in the 1960's, when the observed luminosity of many, if not all, elliptical galaxies could be well fit by truncated isothermals, i.e., the so-called King models. ${ }^{23}$ However, in the intervening years, improved high resolution photometry and better background subtraction have yielded better data for which truncated isothermals do not provide adequate fits. Instead, one is led typically to model data using a de Vaucouleur ${ }^{24}$ density profile or, more recently, a Nuker density law that allows for a central cusp. ${ }^{25}$

Even neglecting the fact that observed galaxies are not well fit by truncated isothermals, the preceding doctrine of violent relaxation is open to several criticisms. Perhaps the most obvious is simply: why should one assume that "most likely" means (essentially) "maximize the Boltzmann entropy"? The usual justification for this standard thermodynamic assumption, and for the combinatoric arguments exploited by Lynden-Bell, simply fails for a system interacting via a long range force like gravity. The standard argument relies crucially on the assumption of approximate extensivity, i.e., the assumption that, if one's system be viewed as a sum of individual pieces, quantities like the total energy are well approximated by a sum of the corresponding quantities for the individual pieces. ${ }^{26}$ Indeed, one could in principle play the same maximization game for any convex phase space functional, ${ }^{27}$ which leads to a natural connection with Casimir arguments from plasma physics. ${ }^{28}$

More pragmatically, one infers from $N$-body simulations that a strongly convulsing mean field potential is not necessary. One observes a comparably efficient approach towards a meta-equilibrium on a time scale $\sim t_{c r}$ both for "violent" evolution, where $\Phi$ exhibits huge changes on very short time scales, and for "nonviolent" evolution, where $\Phi$ exhibits only relatively small changes. ${ }^{29}$ Nonviolent relaxation can be just as efficient as violent relaxation.

Perhaps the most striking feature about violent relaxation is the assumption that the evolution of $\bar{f}$ is completely unconstrained aside from the imposition of some small number of bulk holonomic constraints and coarse-grained conservation of phase. Th This implies seemingly that, when viewed in terms of $\bar{f}$, the flow is chaotic, modulo only a restriction to a suitably defined phase space hypersurface on which bulk quantities like the total energy are constant.

That this assumption is suspect was recognized already when violent relaxation was first proposed, ${ }^{30}$ but at that time it was not possible to implement direct tests computationally. However, during the past fifteen years a number of different workers (cf. Refs. [31-33]) have exploited improved computational resources to test this assumption, and have been led to the conclusion that it is not justified. Specifically, analyses of $N$-body simulations have demonstrated that there is a precise sense in which individual particles tend to "remember"

${ }^{d}$ As Lynden-Bell discussed in an Appendix to his paper, it is straightforward to generalise his derivation of eq. (10), which only conserved the mean field energy (6), to allow for conservation of the mean field angular momentum or any other quantity that can be written as a phase space integral. 
certain aspects of their initial conditions, at least statistically. Thus, e.g., for a variety of different geometries, allowing for both collapse simulations and collisions between galaxies, one discovers that particles that start with small binding energies tend to end up with small binding energies, and visa versa. In the context of the $C B E$, this would seem to indicate that phase space cells with different initial energies do not become "well shuffled."

This effect can be quantified both microscopically and mesoscopically. For example, one can order the particles in a simulation in terms of their binding energies at different times and then compute quantities like the rank correlation $\mathcal{R}(0, t)$ between the initial ordering and the ordering at some later time $t .^{33}$ The net result of such investigations is that, even allowing for an extremely violent evolution, where the virial ratio $2 \mathcal{K} / \mathcal{V}$ changes by factors of two or more within a crossing time $t_{c r}, \mathcal{R}(0, t)>0.6$ at $t=20 t_{c r}$, well after the system appears to have settled down towards a meta-equilibrium.

Alternatively, one can bin the particles in a simulation in terms of their initial binding energies, and then study the properties of the different bins as the system evolves into the future. Here the obvious question is whether, and if so to what extent, the statistical properties of particles in the initial low and high energy bins converge, as would be expected if the particle energies become completely randomized. ${ }^{32,33}$ The conclusion of such an analysis is that, at least over time scales $\ll t_{R}$, the mean energies of the different bins show absolutely no tendency to converge, although the energy dispersions of the different bins do grow.

The seemingly unambiguous conclusion derived from these numerical investigations is that, contrary to what is usually assumed in violent relaxation, the evolution towards a meta-equilibrium can be comparatively nonviolent, and that, even for a violent evolution, particle energies are not totally randomized. One cannot assume a completely shuffled distribution of particle energies. There is thus a need for an alternative theory of "nonviolent relaxation" 32,34 which does not assume a completely chaotic flow.

\section{Alternatives to Violent Relaxation}

When considering a plasma which is displaced only slightly from some meta-equilibrium $f_{0}$, it is customary to visualise evolution in terms of linear Landau damping ${ }^{35}$ which, for simple geometries, implies that perturbations in the density and potential, $\delta \rho$ and $\delta \Phi$, damp exponentially. Physically, this damping is interpreted (cf. Ref. [36]) as reflecting a resonant coupling of (unperturbed) particles with physical velocity $v^{a}$ and a wave (the perturbation) with phase velocity $c^{a}$. Note that this picture necessarily involves a coarsegrained description since it is formulated in terms of configuration space observables like $\rho$ and $\Phi$ rather than the full distribution function $f$.

It is difficult to interpret, let alone implement, Landau's original calculations for systems that are strongly inhomogeneous. However, there is an alternative interpretation which

continues to make sense, namely that Landau damping involves the phase mixing of a superposition of normal modes. In particular, the distinction which galactic dynamicists 
are wont to make (cf. Ref. [2]) between phase mixing and Landau damping is simply not valid. Viewed appropriately, linear Landau damping is a type of phase mixing.

A small perturbation $\delta f$ governed by the Vlasov equation appropriate for a neutral electrostatic plasma will satisfy a linearised evolution equation which can be written symbolically in the form

$$
\frac{\partial \delta f}{\partial t}=\mathcal{A} \delta f
$$

where $\mathcal{A}$ is a linear integro-differential operator. Suppose, however, that the initial perturbation $\delta f(0)$ is expanded in terms of the normal modes of $\mathcal{A}$. If the spectrum of $\mathcal{A}$ is continuous, a smooth initial perturbation necessarily involves a superposition of normal modes, but the evolution of this initial perturbation then implies that quantities like $\delta \rho$ and $\delta \Phi$ necessarily damp exponentially. ${ }^{37}$ In other words, Landau damping is guaranteed. This is intrinsically a phase mixing process, involving the nondissipative spreading of an initial wavepacket. The perturbation manifests an initial coherence that is lost as it evolves into the future. The full $\delta f$ itself does not tend towards zero in any smooth, pointwise sense, but coarse-grained observables like $\delta \rho$ and $\delta \Phi$ do.

Alternatively, the existence of one or more discrete modes implies that there are perturbations which do not phase mix or Landau damp. The classic example thereof is van Kampen ${ }^{38}$ modes, which correspond to waves executing undamped oscillations with a phase velocity $c^{a}$ where resonant coupling to the unperturbed particles is impossible because $f_{0}\left(c^{a}\right) \equiv 0$.

The important point here is that whether or not Landau damping/phase mixing always occurs is determined completely by whether or not the spectrum of $\mathcal{A}$ is purely continuous, and that the specific form of the modes is irrelevant. For this reason, it is possible to prove an analogous result for small perturbations of a gravitational $C B E$ equilibrium. ${ }^{39}$ Specifically, if the modes of the linearised perturbation equation are all continuous, any linearised perturbation will always exhibit Landau damping/phase mixing. If, however, discrete modes exist, there are possible initial perturbations which do not damp.

Determining whether or not the normal modes for realistic $C B E$ equilibria are continuous is extremely difficult. In general it seems impossible to calculate the modes explicitly, and a formal analysis is also hard since $\mathcal{A}$ is not an elliptic operator and evolves a singular integral kernel. It is, however, worth recalling that, at least in a plasma, discrete modes are typically associated with nontrivial boundary conditions, e.g., spatial confinement or the existence of a maximum velocity. Thus, in particular, exponential damping is guaranteed if $f_{0}$ is analytic. However, one can argue that self-gravitating $C B E$ equilibria always have nontrivial boundary conditions. They are always confined to a compact spatial region by the self-consistently determined potential; and there always exists a maximum speed $\left|v_{\max }\left(x^{a}\right)\right|$ since characteristics for which $v$ is too large will quickly escape to infinity.

For this reason, one might reasonably conjecture ${ }^{39}$ that many, but not necessarily all, perturbations will Landau damp. Most initial $\delta \rho$ 's might be expected to damp, but un- 
damped perturbations could exist for modes that manifest coherence on a scale comparable to the size $R_{\text {sys }}$ of the system, or, especially, given nontrivial phase space structures. In this regard, it seems significant that the aforementioned models of galaxies that exhibit undamped oscillations ${ }^{21,22}$ all contain phase space "holes," i.e., regions in the middle of the occupied phase space regions where $f_{0} \rightarrow 0$.

The discussion hitherto has focused on linear Landau damping, i.e., the behaviour of a small initial perturbation which satisfies a linearised evolution equation. However, at least in principle this picture can also be extended to finite amplitude perturbations. In the context of plasma physics, this means considering nonlinear Landau damping, which generalises the linear theory by incorporating the possibility of mode-mode couplings that allow energy transfer between different modes. Because it is very difficult in a realistic gravitational setting to determine the form of the normal modes even for a linearised perturbation, the development of a detailed theory of nonlinear gravitational Landau damping would be extremely difficult. However, despite this problem it is still possible to visualise selfgravitating systems in an identical fashion, even though the forms of the modes are not known explicitly.

It would thus seem reasonable to ask whether it is possible, and fruitful, to interpret the evolution of a generic initial $f(0)$ towards some meta-equilibrium as a manifestation of nonlinear Landau damping. Such a picture, based on a nonlinear evolution driven by mode-mode coupling, is by construction a relatively coherent process, consistent with the statistical regularities observed in $N$-body simulations and significantly less "incoherent" than what is implicit in the standard picture of violent relaxation.

Is there any evidence that this interpretation is actually viable? One reassuring point is that, at least naively, one might expect the time scales to work out correctly. Indeed, by analogy with Landau damping in a plasma, as well as from simple dimensional analysis, one would expect that a perturbation manifesting coherence on a scale $\sim R_{\text {sys }}$ should damp on a time scale of order $t_{c r}$. Strictly speaking, this dimensional argument is only justified for the linear theory, since mode-mode couplings could introduce new time scales related to $t_{c r}$ by ratios of different length scales. However, at least for plasmas one can show that in certain cases allowing for nonlinear mode-mode couplings does not lead to new time scales. ${ }^{40}$

The intuition that dynamical evolution can lead to undamped oscillating modes is of course corroborated by the aforementioned one-dimensional simulations of both gravitational and plasma systems with counter-streaming initial conditions. ${ }^{10}$ Here the late time state involves reasonably large amplitude oscillations, corresponding to nearly periodic variations in the virial ratio $2 \mathcal{K} / \mathcal{V}$ at the $10 \%$ level, characterised by a period $\sim t_{c r}$ and a coherence length $\sim R_{\text {sys }}$. Unlike the exact pulsating solutions, ${ }^{21,22}$ which incorporate true periodicity, the late time state arising in these one-dimensional simulations is not exactly periodic. Rather what one finds is that the non-oscillating, nearly time-independent, component slowly changes as, via phase mixing ("phase wrapping"), it manifests a coarse-grained approach towards a truly time-independent $f_{0}$. 
Another point, not conclusive but arguably suggestive, arises from a consideration of moment codes. These codes approximate the Vlasov equation of plasma physics or the $C B E$ of gravity by a discrete collection of coupled moment equations which have been reduced to a finite set through the introduction of a symplectic trunction (for example, Channell's ${ }^{41}$ BEDLAM code for an electrostatic plasma and the corresponding GADFLI for a self-gravitating system each involve a collection of 203 coupled moments). When used by accelerator dynamicists to study the stability of beam flows, these efficient moment codes have proven spectacularly successful in the sense that they agree with theory and $N$-body simulations (and experiments!), and one might expect analogously that their gravitational analogues could prove powerful tools in diagnosing stability or lack thereof for solutions to the $C B E$.

However, when applied to a system that is far from equilibrium GADFLI (GAlactic Dynamics From Lie-Poisson Integration) yields results that differ markedly from what is observed in $N$-body simulations. ${ }^{42}$ Specifically, GADFLI does not yield the comparatively efficient approach towards equilibrium that is observed in simulations. For example, whereas $N$-body simulations of a collapsing spherical configuration typically exhibit oscillations which damp on a time scale $\sim t_{c r}$, the moment code yields oscillations that only damp appreciably on a significantly longer time scale. The obvious inference is that GADFLI is missing some important physical ingredient that is present in $N$-body codes. One possible interpretation ${ }^{43}$ would be that standard $N$-body codes are contaminated by non-symplectic perturbations that act as a spurious source of dissipation, and that a more satisfactory $N$-body code would exhibit a less efficient approach towards equilibrium. ( $N$-body codes that use a time-centered leap frog scheme are in fact symplectic, but this scheme is not usually used for large $N$ simulations which tend, generically, to exploit variable time steps.) However, a more conservative interpretation is that the $N$-body codes are at least approximately correct, and that GADFLI misses the effects of phase mixing which rely on the existence of a large number - formally an infinite number - of modes.

If one accepts tentatively the hypothesis that the flow associated with a self-gravitating system far from equilibrium should be interpreted in terms of nonlinear Landau damping, one is led to a single, unified picture of an evolution described by the $C B E$ in which the single dominant mechanism is phase mixing/Landau damping. The idea is that, for a generic initial $f(0)$, the approach towards (albeit not to) an equilibrium will involve nonlinear oscillations about some time-independent $f_{0}$, oscillations which may - or may not eventually phase mix away. When the system is far from equilibrium, nonlinearities will be important and mode-mode coupling will play an important role. Eventually, however, as the system approaches equilibrium more closely, nonlinearities will become less important and ordinary linear Landau damping/phase mixing will provide a reasonable description of what is actually happening.

Given this hypothesis, it is also clear what problem one would like ideally to solve: Determine how to break a generic $f(0)$ into two pieces, an equilibrium $f_{0}$ and a correction 
$\delta f(0)$ not necessarily small, such that, at least in the absence of any undamped modes, the perturbation $\delta \rho$ and/or $\delta \Phi$ associated with the evolved $\delta f$ tends to zero at late times.

Another, slightly different, way in which to formulate this same picture, which may provide some insights into how to identify the equilibrium $f_{0}$ lurking in the initial $f(0)$, is the following: A Hamiltonian evolution governed by the $C B E$ corresponds to a flow in an infinitedimensional phase space which is constrained by conservation of phase (i.e., fixed values of all the Casimirs $C$ ) and the fact that the value of the mean field energy $\mathcal{H}$ does not change in time. Imposing these constraints explicitly serves to pick out a (still infinite-dimensional) hypersurface in the phase space to which the flow is restricted. By analogy with finitedimensional Hamiltonian systems (cf. Refs. [44,45]), one might expect that, when evolved into the future, an initial $f(0)$ will exhibit a coarse-grained approach towards some invariant measure on this hypersurface, e.g., towards a suitably defined microcanonical population of the accessible phase space region. If, when viewed in the infinite-dimensional phase space, the flow is chaotic, so that nearby distribution functions $f$ diverge exponentially, one might expect an approach towards an invariant measure which is exponential in time. If, alternatively, the flow is regular, one might instead expect a power law approach. However, in either case it would seem reasonable to expect an approach towards a "phase-mixed" microcanonical distribution proceeding on the natural time scale $t_{c r}$. In this context, the crucial questions would seem to be the following: for the fixed values of $C$ associated with $f(0)$, what equilibrium solutions $f_{0}$ exist, and to what extent can the invariant distribution associated with the evolved $f(0)$ be interpreted as involving (nonlinear) oscillations about some lower energy $f_{0}$ ?

\section{Weak Non-Hamiltonian Corrections to Collisionless Evolution}

This entire picture relies crucially on the fact that the $C B E$ is a Hamiltonian system. That the $C B E$ is Hamiltonian is in turn a manifestation of a very general result, namely that, when suitably interpreted, the mean field description of any Hamiltonian system is itself Hamiltonian. ${ }^{46}$ If, however, any corrections to mean field theory are introduced, one obtains generically a description of the one-particle distribution function $f$ which is no longer Hamiltonian. Physically, this arises because one is treating the full $N$-particle distribution function $\mu$ as being a sum of two "pieces," namely (1) a product of identical one-particle distribution functions $f(i)(i=1, \ldots, N)$ and $(2)$ a correction reflecting particleparticle correlations which are ignored in the mean field description. The point then is that integrating over the correlations to obtain a closed equation for $f$ leads to a nonlocal, non-Hamiltonian evolution equation. ${ }^{47}$

At the level of particle trajectories, this intuition is manifested in the conventional description of binary encounters as resulting in diffusion and dynamical friction. ${ }^{2,6}$ Thus, e.g., for motion in a fixed potential, one can formulate evolution equations of the form

$$
\frac{d x^{a}}{d t}=v^{a} \quad \text { and } \quad \frac{d v_{a}}{d t}=-\frac{\partial \Phi}{\partial x^{a}}-\eta\left(x^{a}, v_{a}\right) v_{a}+\mathcal{F}_{a}
$$


where $\eta$ and $\mathcal{F}_{a}$ are related by a fluctuation-dissipation theorem, in terms of a characteristic "temperature" or mean squared velocity $\Theta$. If, for example, one treats the fluctuating forces as Gaussian white noise with zero mean, $\eta$ must be constant and (cf. Ref. [48])

$$
\left\langle\mathcal{F}_{a}\left(t_{1}\right) \mathcal{F}_{b}\left(t_{2}\right)\right\rangle=2 \Theta \eta \delta_{a b} \delta_{D}\left(t_{1}-t_{2}\right) .
$$

If the friction and noise are weak, they will only cause appreciable changes in the particle energy $E$ (and any other quantities conserved in the Hamiltonian description) on a time scale $\gg t_{c r}$. However, this does not mean that they cannot have appreciable effects on the flow over much shorter time scales. Indeed, they can be important in at least two ways:

1. If the fixed potential is complicated, involving a coexistence of both regular and chaotic orbits, a strictly Hamiltonian evolution can result in chaotic orbits being trapped in certain phase space regions for a comparatively long time, even though, in the $t \rightarrow \infty$ limit, they will diffuse through cantori ${ }^{49}$ or along an Arnold web $^{50}$ to probe a much larger phase space region. ${ }^{51,52}$ However, even very weak friction and noise - so weak that, over short time scales, they have negligible effects on the form of the invariant measure - tend generically to dramatically accelerate this diffusion, thus facilitating a much more rapid approach towards the invariant measure. ${ }^{52-54}$ Thus, e.g., in some cases friction and noise corresponding to a natural time scale as long as $10^{6}-10^{9} t_{c r}$ can have significant statistical effects on the evolution of ensembles of orbits on a time scale as short as $100 t_{c r}$. At least for orbits in a fixed potential, small non-Hamiltonian corrections can greatly facilitate the coarse-grained approach towards a statistical equilibrium.

2. Liouville's Theorem implies that trajectories in a Hamiltonian system cannot selfintersect. Thus, no matter how complicated the potential or the phase space, a Hamiltonian trajectory must evolve in such a fashion as to avoid crossing itself. However, this can lead to complicated microscopic structures, such as, e.g., the "homoclinic tangle" that can arise near separatrices. ${ }^{55}$ The important point then is that even very weak non-Hamiltonian perturbations can break the constraints associated with Liouville's Theorem, thus allowing the tangle to smooth itself out. In other words, small perturbations that break the rigid constraints associated with Liouville's Theorem can serve to fuzz out small scale structures that arise in a purely Hamiltonian description.

These two alternatives have been formulated completely in the context of motion in a fixed potential; and, at the present time, little if anything is really known about the effects of small non-Hamiltonian perturbations in the context of a self-consistent description as provided by the $C B E$. However, by analogy with the simpler problem it would seem reasonable to conjecture that even very weak perturbations that break the constraints associated with conservation of phase could facilitate the approach towards a meta-equilibrium, and that they could help blur the complex structures which can arise microscopically as the system phase mixes towards this meta-equilibrium. 


\section{HOW DOES THE COLLISIONLESS BOLTZMANN EQUATION RELATE TO THE FULL N-BODY PROBLEM?}

The gravitational $\mathrm{N}$-body problem for a collection of particles of comparable mass is chaotic in the sense that a small initial perturbation will typically grow exponentially on

a time scale $\sim t_{c r}$. This phenomenon, long known, ${ }^{56}$ has been studied systematically over the past several years, ${ }^{57-61}$ and the observed behaviour has proven to be extremely robust. Specifically, one finds that the observed exponential growth is largely independent of the detailed choice of initial conditions and initial perturbation. Seemingly independent of the details, small initial perturbations grow exponentially until they become "macroscopic." Thus, in particular, this chaos persists even for a system that is in or near a statistical metaequilibrium. Moreover, one finds that, when scaled in terms of an $N$-dependent crossing time $t_{c r}$, the time scale $\tau$ associated with this instability admits at most a weak dependence on $N$, at least for $N \gg 2$. In particular, the chaos does not appear to turn off for very large $N$. There is no sense in which $\left(\tau / t_{c r}\right) \rightarrow \infty$ for $N \rightarrow \infty$. If anything, the ratio $\tau / t_{c r}$ very slowly decreases with increasing $N .{ }^{60}$

This leads, however, to an important question of principle. The $N$-body problem appears to be chaotic on a time scale $\sim t_{c r}$, but the flow associated with the $C B E$ is often integrable or near-integrable in the sense that many/all of the characteristics are regular, i.e., nonchaotic. So what do the (often near-integrable) $C B E$ characteristics have to do with the true (chaotic) $N$-body problem?

There is no a priori contradiction between a chaotic $N$-body problem and a $C B E$ evolution that is integrable or near-integrable, since the $C B E$ is predicated on the existence of a smooth distribution function $f$ and a smooth potential $\Phi$. However, there is at least one awkward point: Although galactic astronomers teach that $C B E$ characteristics simply track the motion of phase space fluid elements, and that they should not be interpreted as representing the orbits of representative particles, there is oftentimes the implicit idea that, for $N \rightarrow \infty$, true $N$-body orbits will converge towards orbits in the smooth, self-consistent potential, i.e., towards $C B E$ characteristics.

For a singular initial distribution function

$$
f_{N}\left(x^{a}, v_{a}, 0\right)=\sum_{i=1}^{N} m \delta_{D}\left[\mathbf{x}-\mathbf{x}_{i}(0)\right] \delta_{D}\left[\mathbf{v}-\mathbf{v}_{i}(0)\right],
$$

the $C B E$ is of course equivalent to the full $N$-body problem. If, however, one considers a sequence of initial distribution functions with increasing number $N$, decreasing particle mass $m$, and constant $M=N m$, there is no obvious sense in which, for $N \rightarrow \infty$, the initial $f_{N}(0)$ converges towards a smooth distribution function $f_{\infty}(0)$. Rather, what one has is lower amplitude (but still infinite) spikes on progressively shorter scales. It is therefore hard to envision a uniform convergence towards a smooth potential or smooth $C B E$ characteristics.

In this general context, it is also easy to understand qualitatively why chaos might be expected to persist even for very large $N$. Numerical simulations suggest that much, if not 
all, of the chaos observed in the $N$-body problem is associated with "random" interactions between nearest neighbours, perhaps amplified through a coupling to the bulk mean field. If, however, this is true, the time scale associated with the growth of an initial perturbation can be estimated by considering the tidal effects associated with the perturbation of a pair of particles separated by a distance comparable to a typical interparticle spacing. The tidal acceleration associated with a given pair will of course scale as

$$
\delta \ddot{\mathbf{x}}=(\delta \mathbf{x} \cdot \nabla) \mathbf{a} \sim \frac{G m}{r^{3}} \delta \mathbf{x},
$$

where $r$ is their separation and $m$ a typical particle mass. Assuming, however, that $r \sim n^{-1 / 3}$, with $n$ a typical number density, and that, at least roughly, $G N m / R_{s y s} \sim \bar{v}^{2}$ with $\bar{v}$ a typical particle speed, it follows from dimensional analysis that the natural time scale $\tau \sim R_{\text {sys }} / \bar{v} \sim t_{c r}$. As $N$ increases, the mass of the nearest neighbour decreases, but the distance to that neighbour also decreases in a fashion which cancels the effect of decreasing mass.

This leads to another awkward point. $N$-body simulators often "soften" their computations to reduce discreteness effects which, presumably, are more important in the small $N$ systems which can actually be solved than in the real, large $N$ systems which one would like to understand. Thus, e.g., it is customary in direct summation codes to replace the true $1 / r$ kernel of Newtonian gravity by a new $1 / \sqrt{r^{2}+\epsilon^{2}}$ kernel which effectively turns off encounters on scales shorter than the softening length $\epsilon$. However, if $\epsilon$ is chosen to be too large, i.e., not much smaller than the typical interparticle spacing, such a softening effectively kills the chaos which one expects to be present in the honest $N$-body problem even for very large $N$.

The correct answer to the question raised above - how to reconcile a chaotic $N$-body problem with a non-chaotic $C B E$ flow - is not completely clear. What does, however, seem apparent from the preceeding is that, even for very large $N$, true $N$-body trajectories could differ significantly from $C B E$ characteristics.

One interpretation of the $C B E$, which may enable one to finesse this question, is an "ensemble average" interpretation (cf. Ref. $[62,63]$ ), in which any given realisation of the $N$-body problem would be interpreted as involving the evolution of a collection of $N$ initial conditions that randomly sample the smooth distribution function $f(0)$. In this context, there is no reason to expect that the orbits in an $N$-body realisation will closely track characteristics of the $C B E$, but one might at least hope that, in some statistical sense, there is a close correspondence between $N$-body orbits and $C B E$ characteristics.

To help focus on the basic issue, it is useful to consider an interesting thought experiment which, given recent advances in computer hardware, could perhaps actually be performed numerically. Start by specifying a smooth distribution function $f(0)$ and computing the characteristic associated with some phase point $\left(\mathbf{x}_{0}, \mathbf{v}_{0}\right)$. Next sample $f(0)$ to generate many different sets of initial conditions for the $N$-body problem, all with one particle at $\left(\mathbf{x}_{0}, \mathbf{v}_{0}\right)$, and evolve these different sets of initial conditions into the future. The obvious 
question is then: How do the $N$-body orbits generated from $\left(\mathbf{x}_{0}, \mathbf{v}_{0}\right)$ compare with the $C B E$ characteristic, both individually and in terms of statistical averages?

Given the fact that the $N$-body problem is chaotic on a time scale $\sim t_{c r}$, it would seem reasonable to conjecture that the orbits generated in two different $N$-body realisations will diverge exponentially on a time scale $\sim t_{c r}$; and, similarly, one might expect that any given $N$-body orbit will diverge from the $C B E$ characteristic on a comparable time scale. However, one might nevertheless expect that, for sufficiently large $N$, the ensemble average of the different $N$-body orbits generated from the same $\left(\mathbf{x}_{0}, \mathbf{v}_{0}\right)$ will closely track the $C B E$ characteristic for some finite time. In particular, one might conjecture that the rms configuration space deviation between the $N$-body orbits and the $C B E$ characteristic will scale as

$$
\delta r_{r m s}(t) \sim F(N) \exp (t / \tau),
$$

where $\tau \sim t_{c r}$, roughly independent of the total particle number $N$, and where the prefactor $F(N) \rightarrow 0$ for $N \rightarrow \infty$. Thus, e.g., the assumption of random fluctuations associated with a random choice of initial conditions might suggest $F(N) \propto 1 / \sqrt{N}$.

Whether these expectations are correct is not clear. What does, however, seem evident is that galactic astronomers lack a clear understanding of the sense, if any, in which $N$ body orbits coincide approximately with $C B E$ characteristics. This, however, is arguably an important lacuna. Much of the theorist's intuition derives from the study of orbits in smooth potentials presumably associated with $C B E$ equilibria; and much of the standard interpretation of observational data is predicated on the assumption that stars follow simple nonchaotic orbits in a smooth gravitational potential.

\section{ACKNOWLEDGMENTS}

The ideas described here have derived in part from discussions - and arguments - with a number of different individuals, including George Contopoulos, Paul Channell, Salman Habib, Ted Kirkpatrick, Phil Morrison, and Daniel Pfenniger. I am grateful to Tom O'Neil, Dave Merritt, and Richard Lovelace for critical feedback on the talk from which this paper

derives. I am especially grateful to Donald Lynden-Bell for his detailed criticisms of the penultimate version of the manuscript. The final draft was completed while I was a visitor at the Aspen Center for Physics. 


\section{REFERENCES}

1. MiHAlAS, D. \& BINNEY, J. 1981, Galactic Astronomy. Freeman, San Francisco.

2. BINNEY, J. \& TREMAINE, S. 1987. Galactic Dynamics. Princeton University Press, Princeton.

3. SCHWEIZER, F. 1986. Science 231: 227.

4. MAOZ, E. 1991, Astrophys. J. 375: 687.

5. LYNDEN-BELL, D. 1967, Mon. Not. R. Astr. Soc. 136: 101.

6. CHANDRASEKHAR, S. 1943, Principles of Stellar Structure. University of Chicago Press, Chicago.

7. KANDRUP, H. E. 1981, Astrophys. J. 244: 316.

8. HERTEL, P. \& THIRRING, W. 1971. Commun. Math. Phys. 24: 22.

9. RYBICKI, G. 1971. Astrophys. Space Sci. 14: 56.

10. MineaU, P., FEIX, M. R. \& ROUET, J. L. 1990. Astron. Astrophys. 228: 344.

11. LUWEL, M. \& SEVERNE, G. 1985. Astron. Astrophys. 152: 305.

12. YAWN, K. R. \& MILLER, B. N. 1997. Phys. Rev. E, in press.

13. MORRISON, P. J. 1980, Phys. Lett. A 80: 383.

14. KANDRUP, H. E. 1990. Astrophys. J. 351: 104.

15. MORRISON, P. J. \& ELIEZER, S. 1986. Phys. Rev. A 33: 4205.

16. BARTHOLOMEW, P. 1971. Mon. Not. R. Astr. Soc. 151: 333.

17. PFAFFELMOSER, K. 1992. J. Diff. Eqns. 95: 281.

18. SCHAEFFER, J. 1991. Commun. Part. Diff. Eqns. 16: 1313.

19. KORMENDY, J. \& RICHSTONE, D. 1995. Ann. Rev. Astron. Astrophys. 33: 581.

20. BATT, J. 1987. Transport Theory and Statistical Physics 16: 763.

21. LOUIS, P. D. \& GERHART, O. E. 1988. Mon. Not. R. Astr. Soc. 233: 337.

22. SRIDHAR, S. 1989. Mon. Not. R. Astr. Soc. 201: 939.

23. KING, I. 1962, Astron. J. 67: 471.

24. DE VAUCOULEUR, G. 1948, Ann. d'Astrophysique 11: 247.

25. LAUER, T. R., AJHAR, E. A., BYUN, Y.-L., DRESSLER, A., FABER, S. M., GRILLMAIR, C., KORMENDY, J., RICHSTONE, D., \& TREMAINE, S. 1995. Astron. J. 110: 2622.

26. LANDAU, L. D. \& LIFSHITZ, E. M. 1958. Statistical Physics. Pergamon, London.

27. IPSER, J. R. \& HORWITZ, G. 1979. Astrophys. J. 232: 863.

28. HOLM, D. D., MARSDEN, G. E., RATIU, T. \& WEINSTEIN, A. 1985. Phys. Repts. 123: 1 .

29. KANDRUP, H. E., MAHON, M. E., \& SMITH, H. 1993. Astron. Astrophys. 271: 440.

30. CONTOPOULOS, G. 1994. private communication.

31. VAN ALBADA, T. S. 1982, Mon. Not R. Astr. Soc. 201: 939.

32. QUINN, P. J. \& ZUREK, W. H. 1988. Astrophys. J. 331: 1. 
33. KANDRUP, H. E., MAHON, M. E., \& SMITH, H. 1993. Astron. Astrophys. 271; 440.

34. KANDRUP, H. E. 1993. In Advances in Gravitation and Cosmology. B. R. Iyer, A. R. Prasanna, R. K. Varma, and C. V. Vishveshwara, Ed. Wiley Eastern, New Delhi.

35. LANDAU, L. D. 1946. Soviet Phys. - JETP 10: 25.

36. STIX, T. H. 1962, The Theory of Plasma Waves. McGraw-Hill, New York.

37. CASE, K. M. 1959. Ann. Phys. (NY) 7: 349.

38. VAN KAMPEN, N. G. 1955, Physica 21: 949.

39. HABIB, S., KANDRUP, H. E., \& YIP, P. F. 1986, Astrophys. J. 309: 176.

40. MONTGOMERY, D. 1963, Phys. Fluids A 6: 1109.

41. CHANNELL, P. J. 1995. Ann. N. Y. Acad. Sci. 751: 152.

42. KANDRUP, H. E. 1994. Is there Chaos in BEDLAM? University of Florida preprint.

43. CHANNELL, P. J. 1994. private communication.

44. KANDRUP, H. E. \& MAHON, M. E. 1994. Phys. Rev. E 49: 3735.

45. MAHON, M. E., ABERnATHY, R. A., BRADLEY, B. O., \& KANDRUP, H. E. 1995. Mon. Not. R. Astr. Soc. 275: 443.

46. KANDRUP, H. E. 1994. Phys. Rev. D 50: 2425.

47. KUBO, R., TODA, M., \& HASHITSUME, N. 1978, Statistical Physics II. SpringerVerlag, New York.

48. CHANDRASEKHAR, S. 1943. Rev. Mod. Phys. 15: 1.

49. MATHER, J. N. 1982, Topology 21: 45.

50. ARNOLD, V. I. 1964, Russian Math. Surveys 18: 85.

51. CONTOPOULOS, G. 1971. Astron. J. 76: 147.

52. LieBerman, M. A. \& LiChtenBerG, A. J. 1972. Phys. Rev. A 5: 1852.

53. HABIB, S., KANDRUP, H. E., \& MAHON, M. E. 1996. Phys. Rev. E 53: 5473.

54. HABIB, S., KANDRUP, H. E., \& MAHON, M. E. 1997. Astrophys. J., in press.

55. LiCHTENBERG, A. J. \& LIEBERMAN, M. A. 1992, Regular and Chaotic Dynamics. Springer-Verlag, New York.

56. MILLER, R. H. 1964, Astrophys. J. 140: 250.

57. KANDRUP, H. E. \& SMITH, H. 1991. Astrophys. J. 374: 255.

58. KANDRUP, H. E. \& SMITH, H. 1992. Astrophys. J. 386: 635.

59. KANDRUP, H. E., SMITH, H., \& WILlMES, D. E. 1992. Astrophys. J. 399: 627.

60. GOODMAN, J., HEGGIE, D., \& HUT, P. 1994. Astrophys. J. 415: 715.

61. KANDRUP, H. E., MAHON, M. E., \& SMITH, H. 1992. Astrophys. J. 428: 458.

62. OGORODNIKOV, K. F. 1965. Dynamics of Stellar Systems. MacMillan, New York.

63. KLIMONTOVICH, YU. L. 1983, The Kinetic Theory of Electromagnetic Processes. Springer-Verlag, New York.

64. LYNDEN-BELL, D. \& WOOD, R. 1968, Mon. Not. R. Astr. Soc. 138: 495. 